Hence

$$
h_{n+1} \leqq h_{n},
$$

and $h_{n}$ decreases monotonically.

Physical Interpretation.-A physical interpretation of the problem just discussed is given by the small oscillations of a simple pendulum, the cord of which, in Case I, is gradually lengthened, monotonically, remaining finite; while in Case II the cord is monotonically shortened, never becoming less than a positive fixed length.

The same physical picture is useful, too, for showing that the theorem, stated without restriction on $\varphi(t)$, is false. For the way a child swings higher and higher is to lengthen the equivalent simple pendulum on the downward arc, and suddenly to shorten it as the upward arc begins.

Bôcher's theorem $(b), 1$. c., namely that, when $\varphi(t)$ is continuous and

$$
0<g \leqq \varphi(t) \leqq G,
$$

there is one and only one solution which remains finite, and this vanishes at infinity, is correct without any further restriction on the function $\varphi$. An elementary analytic proof can be given by the aid of the law of the mean and Taylor's theorem with the remainder, carried through the term of the second order.

HARVARD UNIVERSITY,

Cambridge, Massachusetts, December 8, 1918.

\title{
PROOF OF A PROPERTY OF THE NORM OF A CYCLOTOMIC INTEGER.
}

BY MR. H. S. VANDIVER.

(Read before the American Mathematical Society April 27, 1918.)

KUMmer in his first proof of the general law of reciprocity between two ideals in a regular cyclotomic algebraic field gave a theorem* which forms a link in his chain of reasoning. Let

* Abhandlungen Berlin Academy, 1859, p. 119, formula (7). 


$$
\begin{aligned}
\omega(x) & =a_{0}+a_{1} x+a_{2} x^{2}+\cdots+a_{p-2} x^{p-2}, \\
\omega & =a_{0}+a_{1} \alpha+\cdots+a_{p-2} \alpha^{p-2},
\end{aligned}
$$

where $a_{0}, a_{1}, \cdots, a_{p-2}$ are integers, $p$ is a prime, $\alpha=e^{2 i \pi / p}$ and $\omega$ is prime to $p$. Write

$$
D_{s}(\omega)=\left[\frac{d^{s} \log \omega\left(e^{v}\right)}{d v^{s}}\right]_{v=0},
$$

and let $N(\omega)$ be the norm of $\omega$. Then we shall prove that

$$
D_{p-1}(\omega) \equiv \frac{\omega(1)^{p-1}-N(\omega)}{p} \quad(\bmod p),
$$

which result was given by Kummer in the article cited. Kummer's proof depended on certain expansions in logarithmic series. In the present paper we proceed from another point of view. Put

$$
N(\omega)=\omega(\alpha) \omega\left(\alpha^{2}\right) \cdots \omega\left(\alpha^{p-1}\right) .
$$

In this relation $\alpha$ may be replaced by an indeterminate $x$ if we add a suitable multiple of $x^{p-1}+x^{p-2}+\cdots+1$. Letting $x=e^{v}$, we may then write

$$
\omega\left(e^{v}\right) \omega\left(e^{2 v}\right) \cdots \omega\left(e^{(p-1) v}\right)=N(\omega)+\frac{e^{v p}-1}{e^{v}-1} X,
$$

where $X$ is a rational integral function of $e^{v}$. Put $v=0$ in this relation; we obtain

$$
p X(1)+N(\omega)=(\omega(1))^{p-1} .
$$

Taking logarithms in (2), differentiating $(p-1)$ times with respect to $v$, and setting $v=0$ we have, if $V=\left(e^{v p}-1\right) /\left(e^{v}-1\right)$,

$$
\begin{aligned}
\left(1+2^{p-1}+3^{p-1}+\cdots+(p-1)^{p-1}\right) D_{p-1}(\omega) & \\
& \equiv\left[\frac{d^{p-2}}{d v^{p-2}}\left(\frac{1}{N+V X} \frac{d(V X)}{d v}\right)\right]_{v=0} \\
& \equiv\left[\frac{1}{N+V X} \frac{d^{p-1}(V X)}{d v^{p-1}}\right]_{v=0}(\bmod p),
\end{aligned}
$$

since $D_{s}(V) \equiv 0(\bmod p)$ for $S<p-1$ and

$$
[N+V X]_{v=0} \neq 0(\bmod p) \text {, }
$$


$\omega$ and therefore $N$ being prime to $p$. We have further

$$
\left[\frac{d^{p-1}(V X)}{d v}\right]_{v=0} \equiv X(1)\left[\frac{d^{p-1} V}{d v}\right]_{v=0} \equiv-X(1)(\bmod p) .
$$

Comparison of (5), (4) and (3) gives the theorem (1).

Philadelphia,

April, 1918.

\section{TRAJECTORIES AND FLAT POINTS ON RULED SURFACES.}

BY MR. J. K. WHITTEMORE.

(Read before the American Mathematical Society April 28 and October $27,1917$.

\$1. Introduction. In the following paper we determine the flat points* of a ruled surface with real rulings, and prove a new property of the orthogonal trajectories of the rulings. This property may be extended to any isogonal trajectory of the rulings, not itself a ruling, and may be regarded as a generalization of Bonnet's familiar theorem. $\dagger$

Let $S$ be a ruled surface with real rulings, $g$ any such ruling, $C$ an orthogonal trajectory of the rulings, generally not a straight line; let the coordinates of any point of $C$ be $x_{0}, y_{0}, z_{0}$, and consider these as functions of $v$, the arc of $C$. When $C$ is not a straight line let the direction cosines of its tangent be $\alpha, \beta, \gamma$, of its principal normal be $l, m, n$, and of its binormal be $\lambda, \mu, \nu$; let $R$ and $T$ be respectively the radii of curvature and torsion of $C, \psi$ the angle measured from the principal normal towards the binormal to the direction chosen as positive on $g$. We suppose $C$ to be a rectifiable curve generally without singular points in the portion considered; the curvature $1 / R$ and the torsion $1 / T$ shall have finite first derivatives with respect to $v$, and $\psi$ shall have a finite second derivative.

The surface $S$ is given by

$$
x=x_{0}+u L, \quad L=l \cos \psi+\lambda \sin \psi,
$$

* Flat points are defined in $\S 4$ of this paper.

$\dagger$ See Eisenhart, Differential Geometry, p. 248. 